\title{
Activation of the CRABPII/RAR pathway by curcumin induces retinoic acid mediated apoptosis in retinoic acid resistant breast cancer cells
}

\author{
PADMAMALINI THULASIRAMAN, GALEN GARRIGA, VEENA DANTHULURI, \\ DANIEL J. McANDREWS and IMRAN Q. MOHIUDDIN
}

Department of Biomedical Sciences, College of Allied Health, University of South Alabama, Mobile, AL 36688, USA

Received September 23, 2016; Accepted February 8, 2017

DOI: $10.3892 /$ or.2017.5495

\begin{abstract}
Due to the anti-proliferative and anti-apoptotic effects of retinoic acid (RA), this hormone has emerged as a target for several diseases, including cancer. However, development of retinoid resistance is a critical issue and efforts to understand the retinoid signaling pathway may identify useful biomarkers for future clinical trials. Apoptotic responses of RA are exhibited through the cellular RA-binding protein II (CRABPII)/retinoic acid receptor (RAR) signaling cascade. Delivery of RA to RAR by CRABPII enhances the transcriptional activity of genes involved in cell death and cell cycle arrest. The purpose of this study was to investigate the role of curcumin in sensitizing RA-resistant triple-negative breast cancer (TNBC) cells to RA-mediated apoptosis. We provide evidence that curcumin upregulates the expression of CRABPII, RAR $\beta$ and RAR $\gamma$ in two different TNBC cell lines. Co-treatment of the cells with curcumin and RA results in increased apoptosis as demonstrated by elevated cleavage of poly(ADP-ribose) polymerase and cleaved caspase-9. Additionally, silencing CRABPII reverses curcumin sensitization of TNBC cells to the apoptotic inducing
\end{abstract}

Correspondence to: Dr Padmamalini Thulasiraman, Department of Biomedical Sciences, College of Allied Health, University of South Alabama, Mobile, AL 36688, USA

E-mail: pthulasiraman@southalabama.edu

Abbreviations: RA, retinoic acid; CRABPII, cellular retinoic acidbinding protein II; RAR, retinoic acid receptor; TNBC, triple-negative breast cancer; PARP, poly(ADP-ribose) polymerase; ER, estrogen receptor; PR, progesterone receptor; HER2, human epidermal growth factor receptor 2; ATRA, all-trans-retinoic acid; PPAR $\beta / \delta$, peroxisome proliferator-activated receptor $\beta / \delta$; FABP5, fatty acid-binding protein 5; GAPDH, glyceraldehyde 3-phosphate dehydrogenase; MTT, 3-(4,5-dimethylthiazol-2-yl)-2,5-diphenyltetrazolium bromide; DMEM, Dulbecco's modified Eagle's medium; FBS, fetal bovine serum; qRT-PCR, quantitative real-time polymerase chain reaction; DMSO, dimethyl sulfoxide

Key words: cellular retinoic acid-binding protein II, retinoic acid receptor, breast cancer, curcumin, retinoic acid effects of RA. These findings provide mechanistic insights into sensitizing TNBC cells to RA-mediated cell death by curcumin-induced upregulation of the CRABPII/RAR pathway.

\section{Introduction}

With an estimated 1.7 million cases of breast cancer diagnosed worldwide in 2012, this type of cancer remains the most common in women. Among the patients with breast cancer, triple-negative breast cancer (TNBC) accounts for $10-20 \%$ of the invasive breast cancer which is defined as estrogen receptor (ER)-negative, progesterone receptor (PR)-negative and human epidermal growth factor receptor 2 (HER2)-negative (1). Due to the lack of markers, it carries poor prognosis and presents an emerging need to understand the biology of this subtype of breast cancer and develop alternative therapeutic options.

Retinoids are structurally related to the hormone of vitamin A with all-trans-retinoic acid (ATRA) being the active metabolite. Despite the toxicity associated with retinoids, it is in general considered to be well tolerated pharmacological agent. Based on the notion that retinoic acid (RA) promotes cell differentiation, regulates proliferation and apoptosis, it has been combined with anthracycline-based chemotherapy to successfully treat acute promyelocytic leukemia with a success rate of $80 \%$ (2). Depending on its interacting partner, RA has distinct biological functions. The ligand binding domain of the retinoic acid receptor (RAR) which includes RAR $\alpha$, $\mathrm{RAR} \beta$ and $\mathrm{RAR} \gamma$ and retinoic $\mathrm{X}$ receptors $\mathrm{RXR} \alpha, \mathrm{RXR} \beta$ and RXR $\gamma$ can interact with RA $(3,4)$ and activate genes that are involved in anti-proliferation, apoptosis, differentiation and cell cycle arrest (5-9). However, binding of RA to the alternative nuclear receptor, peroxisome proliferator-activated receptor $\beta / \delta(\operatorname{PPAR} \beta / \delta)$ transduces signals which facilitate cell growth, promote cell survival and protect cells against apoptosis (10-13). Delivery of RA to RARs is facilitated by cellular RA-binding protein II (CRABPII), which sequesters RA, translocates to the nucleus, channels RA to RAR and enhances the transcriptional activity of RAR target genes $(6,8,14)$. On the other hand, fatty acid-binding protein 5 (FABP5) can transport RA to its cognate receptor, PPAR $\beta / \delta$ which targets genes that 
are involved in proliferation and cell growth (10-13). Due to the dual and opposing function of RA, the growth inhibitory effects of RA are determined by the expression of the regulatory factors, CRABPII, RARs, FABP5 and PPAR $\beta / \delta$. The differential effects of retinoids on anti-proliferation, cell cycle arrest and apoptosis is dependent on the concentration of RA and the time frame of RA treatment $(15,16)$. Retinoids repress genes involved in cell division and cell proliferation, which is followed by differentiation, without affecting cell viability. While decrease in proliferation and cell cycle arrest of cancer cells occurs at an earlier time frame of within 2 days, the apoptotic effects of retinoids begin on day 4 of treatment, with increased apoptosis after day 5 or 6 of treatment $(15,16)$.

Recent study has demonstrated the importance of CRABPII in mammary carcinoma tumor growth suppression through RA-dependent and RA-independent mechanism (17). The clinical significance of CRABPII has been highlighted in several types of cancer, including non-small lung cancer (18), non-myeloma skin cancer (19) and pancreatic cancer cells (20), pinpointing that restoring the CRABPII signaling pathway may serve as a therapeutic intervention to ameliorate the efficacy of RA or sensitize cancer cells to this hormone. While CRABPII acts to deliver RA to its RAR receptors, each of the different isoforms of RAR exhibits specific function with each receptor regulating a subset of distinct genes (21-26). RAR $\beta$ has been implicated in inflammation and tumor suppression (21-23), while the loss of RAR $\gamma$ has been demonstrated in the progression of malignant squamous cell carcinoma $(24,25)$. Selective activation of RAR $\alpha$ by retinoids induces autophagy in ER-positive breast cancer cells (26). Though there may be alternative RA-resistant mechanisms in cancer, the loss of sensitivity to RA in cancers has been related to the lack of CRABPII and RAR expression with increased expression of FABP5 and PPAR $\beta / \delta(11,19,27,28)$.

Phytochemicals have been extensively studied for the treatment of diseases such as cancer. Curcumin, an active ingredient in the dietary spice turmeric (Curcuma Longa), possesses antioxidant, anti-inflammatory and anticancer properties $(29,30)$. The apoptotic effects of curcumin have been observed in several cancers including breast (31), pancreatic (32), prostate (33) and lung (34), while having no cytotoxic effects on healthy cells (35). Despite its low solubility and bioavailability, the combination of curcumin with conventional chemotherapeutic agents has been demonstrated to be effective in cancer regression $(36,37)$. Synthesis of nanocarriers has not only increased the solubility in aqueous solution but has also improved the bioavailability of curcumin towards cancer cells (38-40). Several studies have demonstrated the ability of curcumin to increase the sensitivity of cancer cells to chemotherapeutic drugs $(41,42)$. Through multiple mechanisms, curcumin and its analogs sensitize cancer cells to chemotherapeutic agents, thus overcoming drug resistance and improving susceptibility to growth suppression by conventional drug treatments (43-47).

Previously, we reported that $30 \mu \mathrm{M}$ curcumin sensitizes TNBC cells to RA-mediated growth suppression by altering the expression level of FABP5/PPAR $\beta / \delta$ pathway and targeting PPAR $\beta / \delta$ target genes (45). However, we did not explore the role of curcumin on the second arm of the retinoid pathway, namely CRABPII/RAR, and in this study we evaluated the hypothesis that curcumin mediated upregulation of the CRABPII/RAR pathway in TNBC cells promotes sensitivity to RA-mediated apoptosis. The overall aim of this study is to investigate the effect of curcumin on the CRABPII/RAR pathway and assess the contribution of this pathway in sensitizing TNBC to RA-mediated growth suppression by triggering cell death. In order to achieve this aim, we examine whether regulation of CRABPII/RAR by curcumin is dose-dependent and the functional consequence of this pathway in increasing sensitivity to RA. Furthermore, we explored the impact of silencing CRABPII on apoptosis facilitated by curcumin and/or RA. In the present study, we demonstrate that while $30 \mu \mathrm{M}$ curcumin induces CRABPII, it does not affect RARs in TNBC cells. However, lower doses of curcumin $(5$ and $10 \mu \mathrm{M})$ upregulate CRABPII, RAR $\beta$ and RAR $\gamma$ expression in these cells. We also provide evidence that induction of the CRABPII/RAR pathway by curcumin sensitizes RA-resistant TNBC cells to RA-mediated apoptosis, and knockdown of CRABPII in TNBC cell lines reverses the sensitization of the apoptotic effects of RA by curcumin.

\section{Materials and methods}

Reagents. Antibodies for RAR $\alpha$, RAR $\beta$, RAR $\gamma$ and glyceraldehyde 3-phosphate dehydrogenase (GAPDH) were obtained from Abcam (Cambridge, MA, USA). Anti-CRABPII was purchased from R\&D Systems (Minneapolis, MN, USA). Caspase-9 and poly(ADP-ribose) polymerase (PARP) antibodies were obtained from Cell Signaling Technology, Inc. (Danvers, MA,USA). Anti-mouse and anti-rabbitimmunoglobulin horseradish peroxidase-conjugated antibodies were from Bio-Rad Laboratories, Inc. (Hercules, CA, USA) and anti-goat immunoglobulin was from Santa Cruz Biotechnology, Inc. (Santa Cruz, CA, USA). Curcumin (C-1386) and ATRA (R-2625) were purchased from Sigma-Aldrich (St. Louis, MO, USA). 3-(4,5-Dimethylthiazol-2-yl)-2,5-diphenyltetrazolium bromide (MTT) reagent was purchased from Sigma-Aldrich. CRABPII and control siRNA were obtained from Santa Cruz Biotechnology, Inc.

Cell lines. MDA-MB-231 and MDA-MB-468 cells were maintained in Dulbecco's modified Eagle's medium (DMEM) supplemented with $10 \%$ fetal bovine serum (FBS) with antibiotics. MDA-MB-231 cells were a kind gift from Dr Ming Tan (Mitchell Cancer Institute, Mobile, AL, USA). MDA-MB-468 was purchased from American Type Culture Collection (ATCC; Manassas, VA, USA).

Western blot analyses. Cells were cultured in 100-mm plates and treated with curcumin and/or ATRA in media containing $10 \%$ charcoal treated FBS for the indicated time. Cells were lysed in a buffer containing $150 \mathrm{mM} \mathrm{NaCl}, 10 \mathrm{mM}$ Tris, $\mathrm{pH} 7.2$, $0.1 \%$ SDS, $1 \%$ Triton X-100, $1 \%$ deoxycholate, 5 mM EDTA and $1 \mathrm{mM}$ PMSF for $1 \mathrm{~h}$. The concentration of the whole cell protein was determined using the Bradford assay. Cell lysate was resolved by SDS-PAGE and transferred onto a nitrocellulose membrane. The membrane was blocked in $10 \%$ bovine serum albumin (BSA) in Tris-buffered saline containing $0.05 \%$ Tween-20 for $1 \mathrm{~h}$ at room temperature. The membrane was then probed using the appropriate antibody, CRABPII, 
A

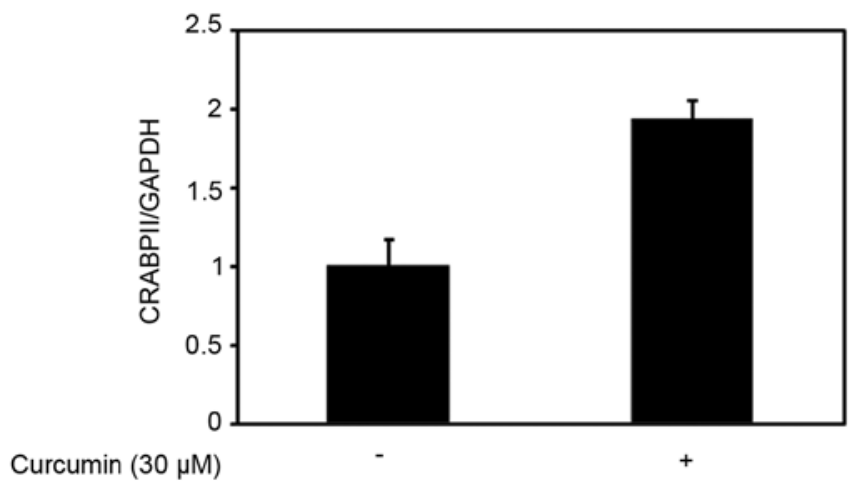

B

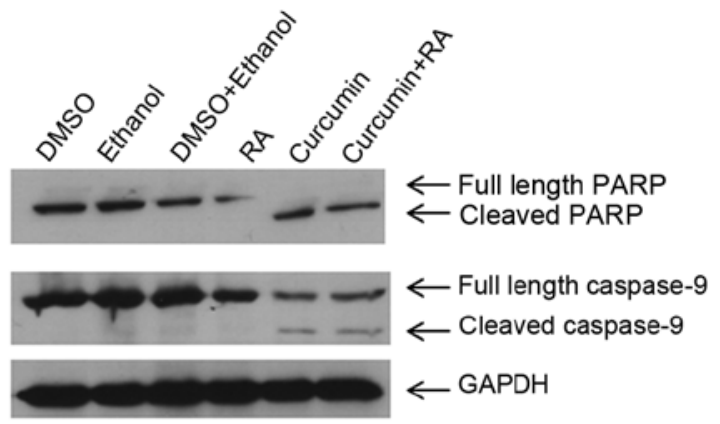

Figure 1. Upregulation of CRABPII by high-dose of curcumin does not sensitize TNBC cells to RA-mediated apoptosis. (A) Total RNA was collected from MDA-MB-231 cells treated with $30 \mu \mathrm{M}$ curcumin for $4 \mathrm{~h}$ (DMSO was used as control). Expression level of CRABPII mRNA in MDA-MB-231 cells was analyzed by qRT-PCR. GAPDH was used for normalization. Data are mean $\pm \mathrm{SE}(\mathrm{n}=3$ ), $\mathrm{p}=0.016$ (curcumin treatment compared to control treatment). (B) MDA-MB-231 cells were treated with $30 \mu \mathrm{M}$ curcumin in the presence or absence of $1 \mu \mathrm{M}$ ATRA (RA) for $48 \mathrm{~h}$ prior to cell lysis. Controls used were DMSO, ethanol or the combination corresponding to the drug treatment. Cell lysates were resolved with SDS-PAGE and immunoblotted with antibodies recognizing PARP and caspase-9. GAPDH was used as a loading control. CRABPII, cellular retinoic acid-binding protein II; TNBC, triple-negative breast cancer; ATRA, all-trans-retinoic acid.

RAR $\alpha, \operatorname{RAR} \beta$, RAR $\gamma$, PARP or caspase-9 at a dilution of 1:1,000 overnight at $4^{\circ} \mathrm{C}$. After a $30 \mathrm{~min}$ wash, the appropriate secondary antibody was added for $1 \mathrm{~h}$ at room temperature prior to exposure. Anti-GAPDH $(1: 2,000)$ was used as a loading control and was incubated with the membrane for $1 \mathrm{~h}$ at $4^{\circ} \mathrm{C}$. The antigen-antibody complex was visualized with SuperSignal West Pico Chemiluminescent Substrate (Thermo Fisher Scientific, Inc., Hanover Park, IL, USA).

Quantitative real-time polymerase chain reaction ( $q R T-P C R)$. Cells were treated with curcumin for $4 \mathrm{~h}$, and RNA was extracted using TRIzol (Life Technologies, Grand Island, NY, USA). As outlined in the protocol for the high-capacity RNA to cDNA kit from Applied Biosystems (Foster City, CA, USA), $2 \mu \mathrm{g}$ total RNA was reverse transcribed into cDNA. To determine expression of CRABPII, RAR $\alpha, \operatorname{RAR} \beta$ and RAR $\gamma$, qRT-PCR was carried out by using commercially available TaqMan chemistry and assay on demand probes (Applied Biosystems). GAPDH was used for normalization. Detection and data analysis were carried out on the ABI StepOnePlus Real-Time PCR system. Relative quantity of gene expression was performed using $2^{-\Delta \Delta C t}$ method (48).

Cell viability assay. MDA-MB-231 and MDA-MB-468 cells $(5,000$ cells/well) were cultured in media containing $10 \%$ charcoal treated FBS and plated in a 96-well plate overnight. Cells were then treated with curcumin in the presence or absence of ATRA for $72 \mathrm{~h}$. Controls used were DMSO and/or ethanol depending on the drug treatment. After $72 \mathrm{~h}$, $5 \mu \mathrm{g} / \mathrm{ml}$ of MTT reagent was added directly to the cells for $3 \mathrm{~h}$ and allowed to incubate at $37^{\circ} \mathrm{C}$. The media was removed from the plate and the intact cells were resuspended in $150 \mu \mathrm{l}$ of $0.04 \mathrm{M} \mathrm{HCl}$ in isopropanol. The cells were placed in the incubator for $15 \mathrm{~min}$ to solubilize the cells, and were then mixed completely. Absorbance was read at $570 \mathrm{~nm}$ to determine cell proliferation.
Cell transfection. MDA-MB-231 and MDA-MB-468 cells were transfected with CRABPII siRNA according to the protocol from Invitrogen Life Technologies (Grand Island, NY, USA). Briefly, 30 pmol of control or CRABPII siRNA was mixed with $500 \mu \mathrm{l}$ Gibco Opti-MEM ${ }^{\circledR}$ I medium without serum by Life Technologies in a 6-well plate. Lipofectamine ${ }^{\mathrm{TM}}$ RNAiMAX $(5 \mu \mathrm{l})$ was added to the diluted RNAi molecules for $20 \mathrm{~min}$ at room temperature. MDA-MB-231 and MDA-MB468 (250,000 cells) were diluted in $2.5 \mathrm{ml}$ of antibiotics-free media and added to the plates containing the RNAi duplex/ Lipofectamine $^{\mathrm{TM}}$ mixture labeled with control or CRABPII siRNA. After $24 \mathrm{~h}$, the cells were treated with $10 \mu \mathrm{M}$ curcumin in the presence or absence of $1 \mu \mathrm{M}$ ATRA for $96 \mathrm{~h}$. ATRA was added every 2 days. Cells were then lysed, protein was extracted, quantitated and protein extract was loaded onto a gel for SDS-PAGE. The blot was probed with the appropriate antibodies.

Statistical analysis. Statistical significance of differences between treatments was determined using two-tailed Student's $\mathrm{t}$-test and P-values were noted. Differences between groups were considered statistically significant at $\mathrm{p}<0.05$.

\section{Results}

Concentration-dependent impact of curcumin on CRABPII and RAR expression in TNBC cell lines. Knowing that TNBC cells are resistant to RA due to the expression patterns of CRABPII and FABP5 $(11,20,49)$, our previous study demonstrated that $30 \mu \mathrm{M}$ curcumin reduced the expression of FABP5 and its cognitive receptor, PPAR $\beta / \delta$ (45). In this study, we sought to examine the effect of curcumin on the CRABPII/RAR pathway in TNBC cells. As shown in Fig. 1A, $30 \mu \mathrm{M}$ curcumin enhanced the CRABPII mRNA expression level compared to control $(\mathrm{p}=0.016)$ in MDA-MB-231 cells, however, had no effect on the RAR isoforms (data not shown). 
A

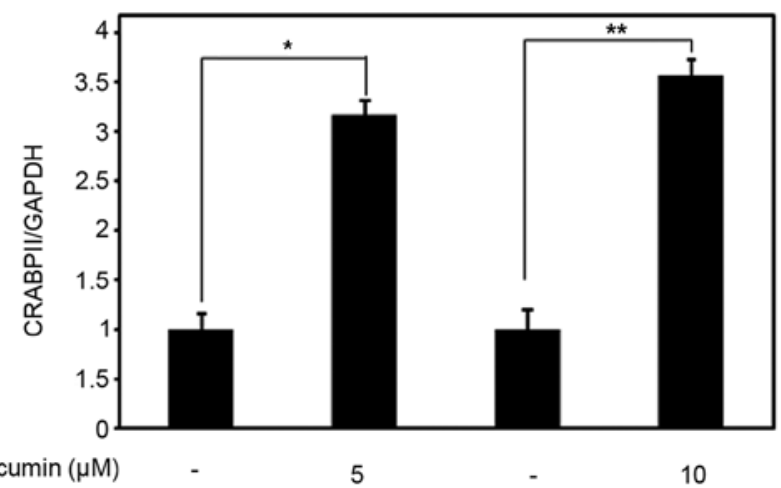

C

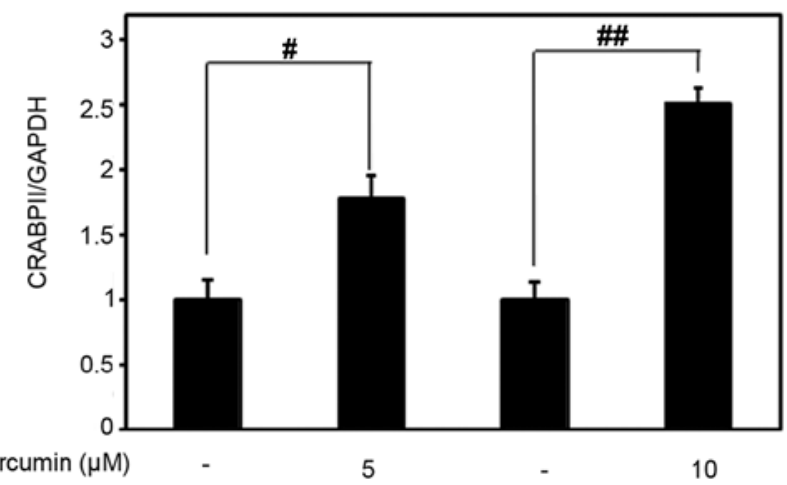

B

Curcumin $(\mu \mathrm{M}) \quad-\quad 5 \quad-\quad 10$

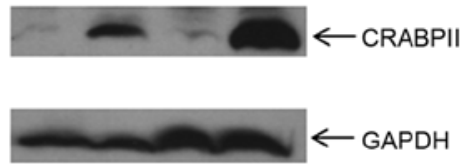

Curcumin $(\mu \mathrm{M})$

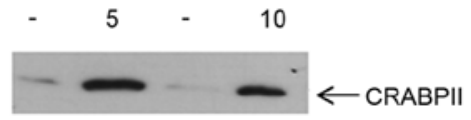

Figure 2. Lower doses of curcumin induce CRABPII expression level in TNBC cell lines. (A) Total RNA was collected from MDA-MB-231 and (C) MDA-MB-468 cells treated with 5 or $10 \mu \mathrm{M}$ curcumin for $4 \mathrm{~h}$ (DMSO was used as control). (A) Expression level of CRABPII mRNA in MDA-MB-231 cells and (C) MDA-MB-468 cells were analyzed by qRT-PCR. GAPDH was used for normalization. Data are mean $\pm \mathrm{SE}(\mathrm{n}=3)$. (A) * $\mathrm{p}=0.005(5 \mu \mathrm{M}$ curcumin treatment compared to the control), ${ }^{* *} \mathrm{p}=0.001$ (10 $\mu \mathrm{M}$ curcumin treatment compared with its respective control). $(\mathrm{C}){ }^{\#} \mathrm{p}=0.047$ (5 $\mu \mathrm{M}$ curcumin treatment compared to the control), ${ }^{\# \#} \mathrm{p}=0.006$ (10 $\mu \mathrm{M}$ curcumin compared with its respective control). (B) MDA-MB-231 and (D) MDA-MB-468 cells were treated with 5 or $10 \mu \mathrm{M}$ curcumin for $24 \mathrm{~h}$ (DMSO was used as control) prior to cell lysis. Cell lysates were resolved with SDS-PAGE and immunoblotted with antibodies recognizing CRABPII, and GAPDH was used as a loading control. CRABPII, cellular retinoic acid-binding protein II; TNBC, triple-negative breast cancer.

Since CRABPII delivers RA to RAR isoforms, we tested whether the induction of CRABPII was sufficient to sensitize RA-resistant MDA-MB-231 cells to RA-mediated apoptosis. To investigate cell death, we tested PARP, an indicator of apoptosis and RAR target, caspase-9. As shown in Fig. 1B, treatment of MDA-MB-231 cells with $30 \mu \mathrm{M}$ curcumin for 48 h completely converted full length PARP to cleaved PARP, and thus there was no effect with the combination of curcumin and RA. Examination of the caspase- 9 showed there was no difference in the expression of cleaved caspase- 9 in cells treated with curcumin compared to the co-treatment with curcumin and ATRA (Fig. 1B). Despite upregulation of CRABPII by $30 \mu \mathrm{M}$ curcumin, this dose of curcumin induced apoptosis in $48 \mathrm{~h}$, independent of the CRABPII pathway.

Because $30 \mu \mathrm{M}$ curcumin is a high-dose which alone induces apoptosis within $48 \mathrm{~h}$, we examined the expression level of CRABPII and RAR isoforms at lower doses of curcumin (5 and $10 \mu \mathrm{M})$. Compared to control, 5 and $10 \mu \mathrm{M}$ curcumin induced CRABPII mRNA expression, with statistical significance $(\mathrm{p}<0.05)$, in MDA-MB-231 cells by 3-3.5-fold (Fig. 2A). Concomitantly, we examined the effect of lower doses of curcumin on CRABPII protein expression. Consistent with the upregulation of CRABPII mRNA expression by 5 and $10 \mu \mathrm{M}$ curcumin, we also observed that at both of these doses, curcumin induced CRABPII protein expression in MDA-MB-231 cells (Fig. 2B). Because curcumin affects
CRABPII expression in RA-resistant MDA-MB-231 cells, we further tested whether curcumin regulates CRABPII mRNA and protein expression in the TNBC cell line, MDA-MB-468. As shown in Fig. 2C, both of the lower doses of curcumin, in comparison to control, upregulated the expression of the CRABPII mRNA $(\mathrm{p}<0.05)$. Correspondingly, curcumin induced CRABPII protein expression level in MDA-MB-468 (Fig. 2D).

We next investigated whether 5 and $10 \mu \mathrm{M}$ curcumin had an effect on the cognate receptors of CRABPII, RAR isoforms $\alpha, \beta$ and $\gamma$. At both of these doses, RAR $\beta$ and RAR $\gamma$ protein expression were upregulated in MDA-MB-231 cells compared to their respective controls (Fig. $3 \mathrm{~A}$ and $\mathrm{B}$ ). To determine whether regulation of $\operatorname{RAR} \beta$ and $\mathrm{RAR} \gamma$ by curcumin is a global effect among TNBC cells, treatment of MDA-MB-468 cells with 5 and $10 \mu \mathrm{M}$ curcumin induced both RAR $\beta$ and RAR $\gamma$ protein expression (Fig. 3C and D). Interestingly, we observed that curcumin did not affect the expression of RAR $\alpha$ in either of the TNBC cell lines, MDA-MB-231 and MDA-MB-468 (data not shown). These results suggest that lower doses of 5 and $10 \mu \mathrm{M}$ curcumin enhance $\mathrm{RAR} \beta$ and $\mathrm{RAR} \gamma$ protein expression in TNBC cells.

Lower doses of curcumin sensitize TNBC cells to RA-mediated apoptosis. Suppression of cell growth by RA is followed by RA-induced apoptosis. Previously, we showed that treatment 
A

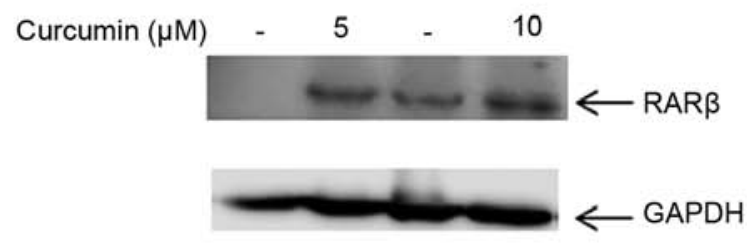

C

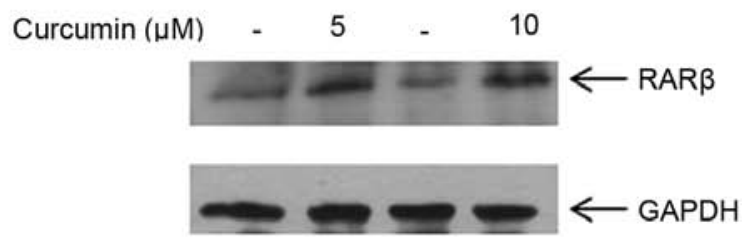

B

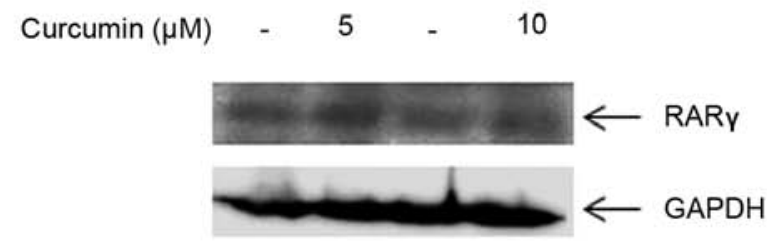

D

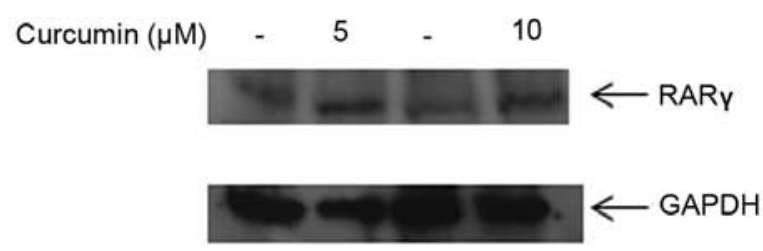

Figure 3. Lower doses of curcumin induce the expression level of RAR $\beta$ and RAR $\gamma$ in TNBC cell lines. (A and B) MDA-MB-231 and (C and D) MDA-MB-468 cells were treated with 5 or $10 \mu \mathrm{M}$ curcumin for $24 \mathrm{~h}$ (DMSO was used for the control) prior to cell lysis. Cell lysates were resolved with SDS-PAGE and immunoblotted with antibodies recognizing RAR $\beta$ and RAR $\gamma$. GAPDH was used as a loading control. RAR, retinoic acid receptor; TNBC, triple-negative breast cancer.

A

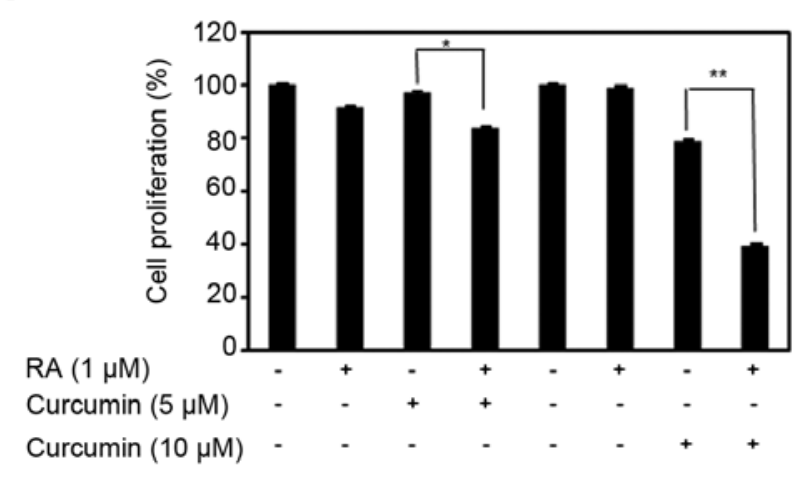

C

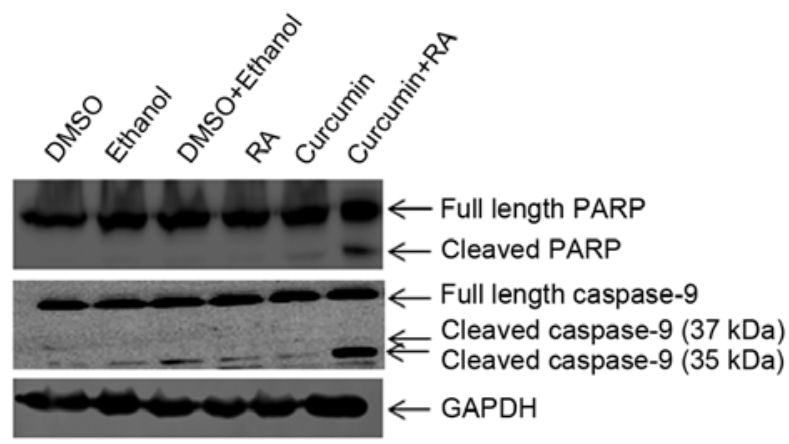

B

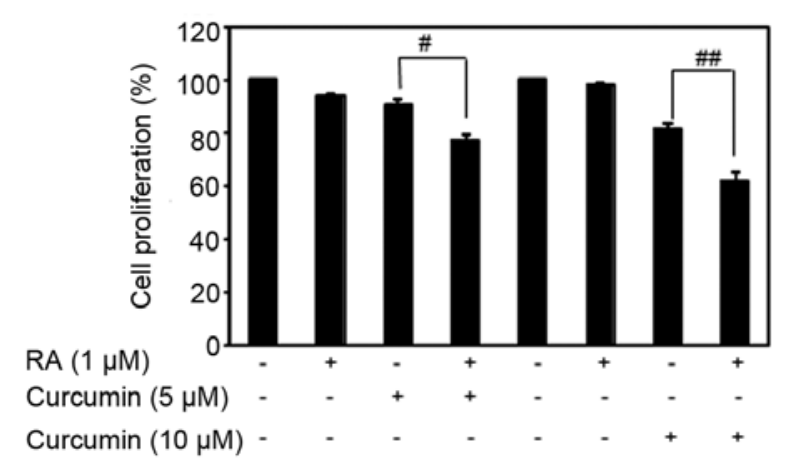

D

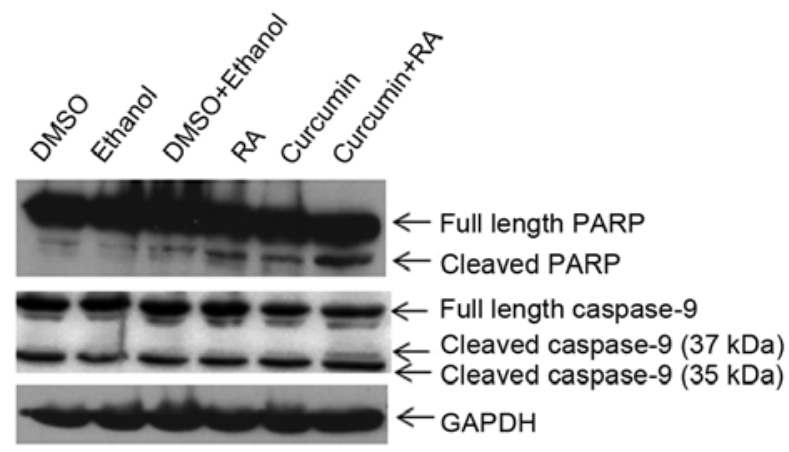

Figure 4. Lower doses of curcumin enhance the sensitivity of TNBC cell lines to RA-mediated growth suppression and apoptosis. (A) MDA-MB-231 and (B) MDA-MB-468 cells were treated with 5 and $10 \mu \mathrm{M}$ curcumin in the presence of absence of $1 \mu \mathrm{M}$ ATRA (RA) for $72 \mathrm{~h}$. The cell proliferation (\%) for each of the treatment was calculated with respect to the controls (DMSO, ethanol or the combination of both). The controls were set at $100 \%$. Data are mean $\pm \mathrm{SE}(\mathrm{n}=3) .{ }^{*} \mathrm{p}=0.037,{ }^{* *} \mathrm{p}=0.04,{ }^{\#} \mathrm{p}=0.0008,{ }^{\# \#} \mathrm{p}=0.012$. (C) MDA-MB-231 and (D) MDA-MB-468 cells were treated with $10 \mu \mathrm{M}$ curcumin in the presence or absence of $1 \mu \mathrm{M}$ ATRA (RA) for $96 \mathrm{~h}$ (DMSO, ethanol or the combination of both was used for control) prior to cell lysis. Cell lysates were resolved with SDS-PAGE and immunoblotted with antibodies recognizing PARP and caspase-9. GAPDH was used as a loading control. TNBC, triple-negative breast cancer.

of TNBC cells with $30 \mu \mathrm{M}$ curcumin for $48 \mathrm{~h}$ sensitized these cells to RA-mediated growth suppression by reducing the expression level of the FABP5/PPAR $\beta / \delta$ pathway (45). In this study, we observed that lower doses of curcumin (5 and $10 \mu \mathrm{M}$ ) induced the expression of CRABPII, RAR $\beta$ and
RAR $\gamma$ (Figs. 2 and 3). To determine the effect of lower doses of curcumin on sensitizing TNBC cells to RA-mediated apoptosis, we treated MDA-MB-231 and MDA-MB-468 cells with 5 and $10 \mu \mathrm{M}$ curcumin in the presence or absence of $1 \mu \mathrm{M}$ ATRA for $72 \mathrm{~h}$. As expected, RA did not inhibit cell 
A

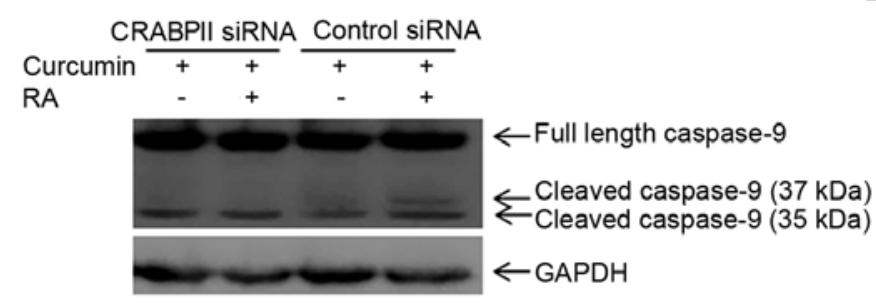

B

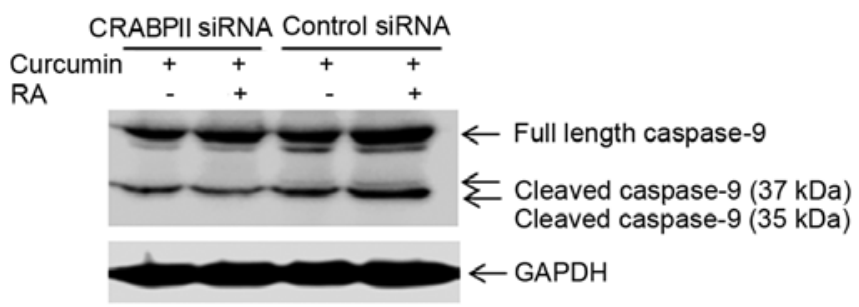

Figure 5. RA-induced apoptosis by curcumin in TNBC cells is regulated by CRABPII/RAR pathway. (A) MDA-MB-231 and (B) MDA-MB-468 cells were transfected with CRABPII or control siRNA for $24 \mathrm{~h}$, then treated with $10 \mu \mathrm{M}$ curcumin in the presence or absence of $1 \mu \mathrm{M}$ ATRA (RA) for $96 \mathrm{~h}$, protein extracted and expression level of caspase-9 was probed with antibodies recognizing caspase-9. GAPDH was used as a loading control. TNBC, triple-negative breast cancer; CRABPII, cellular retinoic acid-binding protein II.

growth in MDA-MB-231 cells (Fig. 4A). While the lower concentration of curcumin $(5 \mu \mathrm{M})$ did not suppress growth of MDA-MB-231 cells after $72 \mathrm{~h}, 10 \mu \mathrm{M}$ curcumin did reduce growth compared to control, with statistical significance ( $\mathrm{p}=0.03$ ) by $\sim 20 \%$ (Fig. 4A). Combining either 5 or $10 \mu \mathrm{M}$ curcumin with ATRA sensitized MDA-MB-231 cells to RA-mediated growth suppression, with a robust effect with $10 \mu \mathrm{M}$ curcumin and ATRA (Fig. 4A). Similar findings were concluded in MDA-MB-468 cells. RA treatment had marginal growth inhibitory effects on MDA-MB-468 cells $(\mathrm{p}<0.05)$. In comparison to control treated cells, lower doses of curcumin (5 and $10 \mu \mathrm{M}$ ) statistically reduced growth of MDA-MB-468 cells ( $\mathrm{p}<0.05$ ) but the combination of curcumin with RA sensitized MDA-MB-468 cells to RA-induced growth suppression, with a more pronounced effect with $10 \mu \mathrm{M}$ curcumin (Fig. 4B).

Knowing that $10 \mu \mathrm{M}$ curcumin had a greater impact on sensitization of TNBC cells to RA-induced growth suppression at $72 \mathrm{~h}$ (Fig. 4A and B), and the fact that $10 \mu \mathrm{M}$ curcumin induced CRABPII, RAR $\beta$ and RAR $\gamma$ expression in these cells (Figs. 2 and 3), we tested whether this dose of curcumin could sensitize TNBC cells to RA-mediated apoptosis. Because the apoptotic effects of RA occur subsequent to growth suppression, we examined cell death by curcumin and RA at a later time-point. Cells were treated with $10 \mu \mathrm{M}$ curcumin in the presence or absence of $1 \mu \mathrm{M}$ ATRA for $96 \mathrm{~h}$ and PARP expression was examined. In MDA-MB-231 cells, RA did not activate PARP cleavage, while $10 \mu \mathrm{M}$ curcumin potentiated cleaved PARP (Fig. 4C). Co-treatment of curcumin and RA further enhanced active, cleaved PARP compared to curcumin treatment alone. Activation of RAR induces the transcriptional activity of several downstream targets, such as apoptotic protein caspase-9. Concurrently, we examined the effect of curcumin and RA on caspase- 9 expression in MDA-MB-231 cells. As shown in Fig. 4C, curcumin alone marginally induced cleaved caspase-9, while the combination of curcumin with RA enhanced the expression of cleaved/active caspase-9. Similarly, the effects of ATRA and/or curcumin on the activation of PARP and caspase- 9 was examined in MDA-MB-468 cells. The combination of curcumin and RA further enhanced apoptosis as evidenced by increased cleaved PARP in comparison to curcumin or RA alone (Fig. 4D). In addition, the treatment of MDA-MB-468 cells with curcumin and RA induced cleavage of caspase-9 $(37 \mathrm{kDa})$ and reduced inactive caspase- 9 expression compared to either treatment
(Fig. 4D). Results from the data demonstrate the ability of curcumin to potentiate the apoptotic effects of RA and reverse RA-resistance in TNBC by curcumin-mediated induction of CRABPII and RARs.

Sensitization to RA-mediated apoptosis by curcumin is regulated by CRABPII. Since CRABPII/RAR is involved in RA-mediated cell death, we sought to investigate whether upregulation of CRABPII by curcumin (Fig. 2) regulates RA-induced apoptosis. To test this hypothesis, we silenced CRABPII expression in MDA-MB-231 cells, treated the cells with $10 \mu \mathrm{M}$ curcumin in the presence or absence of $1 \mu \mathrm{M}$ ATRA for $96 \mathrm{~h}$ and examined for the protein expression of caspase-9. MDA-MB-231 transfected with control siRNA was also treated with curcumin and as expected, curcumin induced apoptosis by activation of caspase-9 (37 kDa), which was further enhanced in the presence of RA (Fig. 5A). However, when the cells were transfected with CRABPII siRNA, the combination of curcumin and RA abolished the active $37 \mathrm{kDa}$ form of caspase-9 (Fig. 5A). These results suggest that curcumin mediated upregulation of CRABPII sensitizes TNBC cells to RA-mediated apoptosis by the induction of caspase-9, one of the RAR target genes. We also silenced CRABPII in MDA-MB-468 cells and combinatorial treatment of the cells with curcumin and RA enhanced expression of cleaved/active caspase-9 (37 and $35 \mathrm{kDa}$ ), in comparison to curcumin alone (Fig. 5B). However, knockdown of CRABPII reduced active caspase- $9(35 \mathrm{kDa})$ and abolished the expression of the cleaved/active $37 \mathrm{kDa}$ caspase-9 (Fig. 5B).

\section{Discussion}

Sensitivity of cancer cells to RA is determined by two distinct pathway, CRABPII/RAR and FABP5/PPAR $\beta / \delta(10,11,14$, $20,49)$. While the delivery of RA to the FABP5/PPAR $\beta / \delta$ pathway results in increased proliferation, transporting RA by CRABPII to RAR inhibits proliferation and promotes apoptosis $(10,11,14)$. Reducing the ratio of FABP5:CRABPII can overcome the resistance of cancer cells to RA by shifting the delivery of RA to RAR by CRABPII instead of activating the FABP5/PPAR $\beta / \delta$ pathway $(10,11,14)$.

Phytochemicals such as curcumin exhibit chemosensitizing properties which may circumvent toxicity issues faced with traditional chemotherapeutic agents (50). While they are less toxic and have no side effects, combinatorial treatment 
of phytochemicals with chemotherapeutic agents can be an alternative method to reduce the dosage of traditional chemotherapeutic regimens and lower cardiac toxicity associated with them (50). Owing to drug resistance in cancer, curcumin has been shown to be an effective adjuvant in reversing chemoresistance and sensitizing cancer cells to chemotherapeutic drugs (41-47,51-54).

Our previous study reported that the potential therapeutic activity of curcumin to sensitize RA-resistant TNBC cells to RA-mediated growth suppression was exhibited through the inhibition of the FABP5/PPAR $\beta / \delta$ pathway (45). The present study focused on the effect of curcumin on the CRABPII/RAR pathway to reverse resistance of TNBC cells to RA by activation of apoptosis. Results of the present study demonstrate that low concentrations of 5 and $10 \mu \mathrm{M}$ curcumin increase the expression level of CRABPII, while concomitantly induces expression of its cognate receptor, RAR $\beta$ and RAR $\gamma$. Similarly, a recent study demonstrated that curcumin upregulated the expression of RAR $\beta$ in several cancer cells, including TNBC MDA-MB-231 cells and activated RAR $\beta$ via epigenetic regulation (22). Though the RAR subtypes have different functions and regulate distinct RARE targeted genes, RAR signaling plays a critical role in cancer progression. While $\operatorname{RAR} \beta$ is epigenetically silenced in tumor cells and its loss has been associated with lymph node metastasis $(21,55,56), \operatorname{RAR} \gamma$ has been implicated as a tumor suppressor and restoration of this retinoid receptor reverses the tumorigenic potential of mouse keratinocytes $(57,58)$. While curcumin induces the expression of several regulatory genes involved in apoptosis (59), we present evidence in this study that curcumin upregulates genes involved in the retinoid signaling pathway, namely CRABPII, RAR $\beta$ and RAR $\gamma$ in mammary carcinoma MDA-MB-231 and MDA-MB-468 cells.

Although $30 \mu \mathrm{M}$ of curcumin induces the mRNA expression level of CRABPII, it did not affect the expression of RARs. However, lower doses of curcumin (5 and $10 \mu \mathrm{M})$ induces the protein expression level of not only CRABPII but also RAR $\beta$ and RAR $\gamma$ in two TNBC cell lines suggesting that this is a global effect among TNBC cells. Alterations in gene expression associated with different doses of curcumin are not unusual and have been well documented with other genes (60). The differential effects of curcumin have been observed with several apoptotic genes that are upregulated or downregulated by only higher dose of curcumin, while the reverse has also been observed for some genes that are upregulated only at lower doses of curcumin (60). As noted in this study, the differences in concentration of curcumin to induce the CRABPII/RAR pathway may be an important determinant in identifying the required optimum dosage to sensitize TNBC cells to RA for future in vivo studies.

The concentration-dependent regulation of CRABPII and RARs by curcumin determined the outcome on the activation of apoptotic proteins, PARP and caspase-9. Although $30 \mu \mathrm{M}$ curcumin upregulates the mRNA level of CRABPII, this dose of curcumin completely activates PARP and induces activation of caspase-9 in $48 \mathrm{~h}$. However, the fact that $30 \mu \mathrm{M}$ curcumin does not regulate the RARs indicates that this concentration of curcumin induces apoptosis independent of the CRABPII/RAR and $30 \mu \mathrm{M}$ curcumin does not sensitize MDA-MB-231 cells to RA-induced apoptosis.
Curcumin has a differential effect on gene regulation and cell death initiated by this agent dose- and time-dependently $(60,61)$. The present data demonstrates that curcumin can re-activate the CRABPII/RAR pathway in TNBC cells and cause RA to initiate apoptosis by activation of PARP and caspase-9. Such doses of curcumin ( 5 and $10 \mu \mathrm{M})$ upregulates $\operatorname{RAR} \beta$ and RAR $\gamma$, as well as CRABPII in TNBC cells. Combination of $10 \mu \mathrm{M}$ curcumin with RA for $96 \mathrm{~h}$ sensitizes TNBC cells to apoptosis mediated by RA as evidenced by increased PARP cleavage. Because $10 \mu \mathrm{M}$ curcumin induces RAR $\beta$ and RAR $\gamma$ in TNBC cells, curcumin at this dose sensitizes the cells to RA-mediated apoptosis through RAR-dependent activation of caspase-9. Initiation of cell death by RAR itself is not sufficient to regulate apoptosis by RA, and hence shuttling of RA from the cytosol to the nucleus by CRABPII facilitates binding of RA to RARs and enhances the transcriptional activation of genes such as caspase-9 involved in the retinoid signaling pathway. To extend these studies and gain a mechanistic understanding on the role of curcumin on the CRABPII/RAR pathway, our results also provide evidence that silencing CRABPII prevents curcumin from sensitizing TNBC cells to RA-induced activation of caspase-9. Taken together, our data suggest that in order to activate cell death by RA in RA-resistant TNBC cells, CRABPII and RAR pathway have to be upregulated by lower concentrations of curcumin and these two proteins work in concert to sensitize cells to RA-mediated apoptosis.

In conclusion, the present study revealed that reversing the resistance of TNBC to RA-induced apoptosis is dependent on the dose of curcumin and length of treatment. Accordingly, lower concentrations of curcumin induce CRABPII, RAR $\beta$ and RAR $\gamma$, and thus upregulation of CRABPII/RAR pathway contributes to the sensitization of TNBC cells to apoptosis by RA. As such this study highlights a novel mechanism by which RA-resistant mammary carcinoma cells can be resensitized to RA-mediated apoptosis by curcumin. The effectiveness in the combination of curcumin with RA warrants further consideration for its use in RA-resistant TNBC cells. Overall, this study provides mechanistic insights on the role of curcumin to reverse RA resistance in breast cancer cells through the regulation of the CRABPII/RAR pathway, and highlights the potential of using curcumin as a therapeutic adjuvant in RA resistant cancers.

\section{Acknowledgements}

This study was supported by the Research and Scholarship Development Grant Program, University of South Alabama, Office of Research and Economic Development and the start-up funds from the College of Allied Health Professions at University of South Alabama. We thank the Department of Pharmacology, University of South Alabama for use of their film developer.

\section{References}

1. Boyle P: Triple-negative breast cancer: epidemiological considerations and recommendations. Ann Oncol 23 (Suppl 6): vi7-vi12, 2012.

2. Lo-Coco F, Avvisati G, Vignetti M, Thiede C, Orlando SM, Iacobelli S, Ferrara F, Fazi P, Cicconi L, Di Bona E, et al; Gruppo Italiano Malattie Ematologiche dell'Adulto; German-Austrian Acute Myeloid Leukemia Study Group; Study Alliance Leukemia: Retinoic acid and arsenic trioxide for acute promyelocytic leukemia. N Engl J Med 369: 111-121, 2013. 
3. Chambon P: A decade of molecular biology of retinoic acid receptors. FASEB J 10: 940-954, 1996.

4. Germain P, Chambon P, Eichele G, Evans RM, Lazar MA, Leid M, De Lera AR, Lotan R, Mangelsdorf DJ and Gronemeyer H: International Union of Pharmacology. LXIII. Retinoid X receptors. Pharmacol Rev 58: 760-772, 2006

5. Koeffler HP: Is there a role for differentiating therapy in non-APL AML? Best Pract Res Clin Haematol 23: 503-508, 2010.

6. Donato LJ, Suh JH and Noy N: Suppression of mammary carcinoma cell growth by retinoic acid: the cell cycle control gene Btg2 is a direct target for retinoic acid receptor signaling. Cancer Res 67: 609-615, 2007.

7. Altucci L, Rossin A, Raffelsberger W, Reitmair A, Chomienne C and Gronemeyer H: Retinoic acid-induced apoptosis in leukemia cells is mediated by paracrine action of tumor-selective death ligand TRAIL. Nat Med 7: 680-686, 2001.

8. Donato LJ and Noy N: Suppression of mammary carcinoma growth by retinoic acid: proapoptotic genes are targets for retinoic acid receptor and cellular retinoic acid-binding protein II signaling. Cancer Res 65: 8193-8199, 2005.

9. Kitareewan S, Pitha-Rowe I, Sekula D, Lowrey CH, Nemeth MJ, Golub TR, Freemantle SJ and Dmitrovsky E: UBE1L is a retinoid target that triggers PML/RARalpha degradation and apoptosis in acute promyelocytic leukemia. Proc Natl Acad Sci USA 99: 3806-3811, 2002.

10. Schug TT, Berry DC, Shaw NS, Travis SN and Noy N: Opposing effects of retinoic acid on cell growth result from alternate activation of two different nuclear receptors. Cell 129: 723-733, 2007.

11. Schug TT, Berry DC, Toshkov IA, Cheng L, Nikitin AY and Noy N: Overcoming retinoic acid-resistance of mammary carcinomas by diverting retinoic acid from PPARbeta/delta to RAR. Proc Natl Acad Sci USA 105: 7546-7551, 2008.

12. Tan NS, Shaw NS, Vinckenbosch N, Liu P, Yasmin R, Desvergne B, Wahli W and Noy N: Selective cooperation between fatty acid binding proteins and peroxisome proliferator-activated receptors in regulating transcription. Mol Cell Biol 22: 5114-5127, 2002.

13. Di-Poï N, Tan NS, Michalik L, Wahli W and Desvergne B Antiapoptotic role of PPARbeta in keratinocytes via transcriptional control of the Akt1 signaling pathway. Mol Cell 10: 721-733, 2002.

14. Wolf G: Cellular retinoic acid-binding protein II: a coactivator of the transactivation by the retinoic acid receptor complex RAR. RXR. Nutr Rev 58: 151-153, 2000.

15. El-Metwally TH, Hussein MR, Pour PM, Kuszynski CA and Adrian TE: Natural retinoids inhibit proliferation and induce apoptosis in pancreatic cancer cells previously reported to be retinoid resistant. Cancer Biol Ther 4: 474-483, 2005.

16. El-Metwally TH, Hussein MR, Pour PM, Kuszynski CA and Adrian TE: High concentrations of retinoids induce differentiation and late apoptosis in pancreatic cancer cells in vitro. Cancer Biol Ther 4: 602-611, 2005.

17. Vreeland AC, Levi L, Zhang W, Berry DC and Noy N: Cellular retinoic acid-binding protein 2 inhibits tumor growth by two distinct mechanisms. J Biol Chem 289: 34065-34073, 2014.

18. Favorskaya I, Kainov Y, Chemeris G, Komelkov A, Zborovskaya I and Tchevkina E: Expression and clinical significance of CRABP1 and CRABP2 in non-small cell lung cancer. Tumour Biol 35: 10295-10300, 2014.

19. Passeri D, Doldo E, Tarquini C, Costanza G, Mazzaglia D, Agostinelli S, Campione E, Di Stefani A, Giunta A, Bianchi L, et al: Loss of CRABP-II characterizes human skin poorly differentiated squamous cell carcinomas and favors DMBA/TPA-induced carcinogenesis. J Invest Dermatol 136 : $1255-1266,2016$

20. Gupta S, Pramanik D, Mukherjee R, Campbell NR, Elumalai S, de Wilde RF, Hong SM, Goggins MG, De Jesus-Acosta A, Laheru D, et al: Molecular determinants of retinoic acid sensitivity in pancreatic cancer. Clin Cancer Res 18: 280-289, 2012.

21. Albino-Sanchez ME, Vazquez-Hernandez J, Ocadiz-Delgado R, Serafin-Higuera N, León-Galicia I, Garcia-Villa E, Hernandez-Pando R and Gariglio P: Decreased RAR $\beta$ expression induces abundant inflammation and cervical precancerous lesions. Exp Cell Res 346: 40-52, 2016.

22. Jiang A, Wang X, Shan X, Li Y, Wang P, Jiang P and Feng Q: Curcumin reactivates silenced tumor suppressor gene RAR $\beta$ by reducing DNA methylation. Phytother Res 29: 1237-1245, 2015.

23. Xu XC: Tumor-suppressive activity of retinoic acid receptor-beta in cancer. Cancer Lett 253: 14-24, 2007.
24. Darwiche N, Celli G, Tennenbaum T, Glick AB, Yuspa SH and De Luca LM: Mouse skin tumor progression results in differential expression of retinoic acid and retinoid X receptors. Cancer Res 55: 2774-2782, 1995.

25. Xu XC, Wong WY, Goldberg L, Baer SC, Wolf JE, Ramsdell WM, Alberts DS, Lippman SM and Lotan R: Progressive decreases in nuclear retinoid receptors during skin squamous carcinogenesis. Cancer Res 61: 4306-4310, 2001.

26. Brigger D, Schläfli AM, Garattini E and Tschan MP: Activation of RAR $\alpha$ induces autophagy in SKBR3 breast cancer cells and depletion of key autophagy genes enhances ATRA toxicity. Cell Death Dis 6: e1861,2015.

27. Tomita A, Kiyoi H and Naoe T: Mechanisms of action and resistance to all-trans retinoic acid (ATRA) and arsenic trioxide $\left(\mathrm{As}_{2} \mathrm{O}_{3}\right)$ in acute promyelocytic leukemia. Int J Hematol 97: 717-725, 2013.

28. Applegate CC and Lane MA: Role of retinoids in the prevention and treatment of colorectal cancer. World J Gastrointest Oncol 7: 184-203, 2015

29. Ak T and Gülçin I: Antioxidant and radical scavenging properties of curcumin. Chem Biol Interact 174: 27-37, 2008.

30. Joe B, Vijaykumar M and Lokesh BR: Biological properties of curcumin-cellular and molecular mechanisms of action. Crit Rev Food Sci Nutr 44: 97-111, 2004.

31. Wang Y, Yu J, Cui R, Lin J and Ding X: Curcumin in treating breast cancer (Review). J Lab Autom 21: 723-731, 2016.

32. Bimonte S, Barbieri A, Leongito M, Piccirillo M, Giudice A, Pivonello C, de Angelis C, Granata V, Palaia R and Izzo F: Curcumin anticancer studies in pancreatic cancer. Nutrients 8: $8,2016$.

33. Jordan BC, Mock CD, Thilagavathi R and Selvam C: Molecular mechanisms of curcumin and its semisynthetic analogues in prostate cancer prevention and treatment. Life Sci 152: 135-144, 2016.

34. Howells LM, Mahale J, Sale S, McVeigh L, Steward WP, Thomas A and Brown K: Translating curcumin to the clinic for lung cancer prevention: evaluation of the preclinical evidence for its utility in primary, secondary, and tertiary prevention strategies. J Pharmacol Exp Ther 350: 483-494, 2014.

35. Chainani-Wu N: Safety and anti-inflammatory activity of curcumin: a component of tumeric (Curcuma longa). J Altern Complement Med 9: 161-168, 2003.

36. Cheah YH, Nordin FJ, Sarip R, Tee TT, Azimahtol HL, Sirat HM, Rashid BA, Abdullah NR and Ismail Z: Combined xanthorrhizol-curcumin exhibits synergistic growth inhibitory activity via apoptosis induction in human breast cancer cells MDA-MB-231. Cancer Cell Int 9: 1, 2009.

37. Javvadi P, Segan AT, Tuttle SW and Koumenis C: The chemopreventive agent curcumin is a potent radiosensitizer of human cervical tumor cells via increased reactive oxygen species production and overactivation of the mitogen-activated protein kinase pathway. Mol Pharmacol 73: 1491-1501, 2008.

38. Anitha A, Maya S, Deepa N, Chennazhi KP, Nair SV and Jayakumar R: Curcumin-loaded N,O-carboxymethyl chitosan nanoparticles for cancer drug delivery. J Biomater Sci Polym Ed 23: 1381-1400, 2012

39. Mukerjee A and Vishwanatha JK: Formulation, characterization and evaluation of curcumin-loaded PLGA nanospheres for cancer therapy. Anticancer Res 29: 3867-3875, 2009.

40. Ma Z, Haddadi A, Molavi O, Lavasanifar A, Lai R and Samuel J: Micelles of poly(ethylene oxide)-b-poly(epsilon-caprolactone) as vehicles for the solubilization, stabilization, and controlled delivery of curcumin. J Biomed Mater Res A 86: 300-310, 2008.

41. Bordoloi D, Roy NK, Monisha J, Padmavathi G and Kunnumakkara AB: Multi-targeted agents in cancer cell chemosensitization: what we learnt from curcumin thus far. Recent Patents Anticancer Drug Discov 11: 67-97, 2016.

42. Saha S, Adhikary A, Bhattacharyya P, Das T and Sa G: Death by design: where curcumin sensitizes drug-resistant tumours. Anticancer Res 32: 2567-2584, 2012.

43. Chen P, Li J, Jiang HG, Lan T and Chen YC: Curcumin reverses cisplatin resistance in cisplatin-resistant lung caner cells by inhibiting FA/BRCA pathway. Tumour Biol 36: 3591-3599, 2015

44. Toden S, Okugawa Y, Jascur T, Wodarz D, Komarova NL, Buhrmann C, Shakibaei M, Boland CR and Goel A: Curcumin mediates chemosensitization to 5-fluorouracil through miRNA-induced suppression of epithelial-to-mesenchymal transition in chemoresistant colorectal cancer. Carcinogenesis 36: 355-367, 2015. 
45. Thulasiraman P, McAndrews DJ and Mohiudddin IQ: Curcumin restores sensitivity to retinoic acid in triple negative breast cancer cells. BMC Cancer 14: 724, 2014.

46. Zhou B, Huang J, Zuo Y, Li B, Guo Q, Cui B, Shao W, Du J and $\mathrm{Bu} \mathrm{X}$ : 2a, a novel curcumin analog, sensitizes cisplatin-resistant A549 cells to cisplatin by inhibiting thioredoxin reductase concomitant oxidative stress damage. Eur J Pharmacol 707: 130-139, 2013.

47. Jiang M, Huang O, Zhang X, Xie Z, Shen A, Liu H, Geng M and Shen K: Curcumin induces cell death and restores tamoxifen sensitivity in the antiestrogen-resistant breast cancer cell lines MCF-7/LCC2 and MCF-7/LCC9. Molecules 18: 701-720, 2013.

48. Livak KJ and Schmittgen TD: Analysis of relative gene expression data using real-time quantitative PCR and the 2[-Delta Delta C(T)] method. Methods 25: 402-408, 2001.

49. Liu RZ, Graham K, Glubrecht DD, Germain DR, Mackey JR and Godbout R: Association of FABP5 expression with poor survival in triple-negative breast cancer: implication for retinoic acid therapy. Am J Pathol 178: 997-1008, 2011.

50. Prasad NR, Muthusamy G, Shanmugam M and Ambudkar SV: South Asian medicinal compounds as modulators of resistance to chemotherapy and radiotherapy. Cancers (Basel) 8: 8, 2016.

51. Montgomery A, Adeyeni T, San K, Heuertz RM and Ezekiel UR Curcumin sensitizes silymarin to exert synergistic anticancer activity in colon cancer cells. J Cancer 7: 1250-1257, 2016.

52. Ruiz de Porras V, Bystrup S, Martínez-Cardús A, Pluvinet R, Sumoy L, Howells L, James MI, Iwuji C, Manzano JL, Layos L, et al: Curcumin mediates oxaliplatin-acquired resistance reversion in colorectal cancer cell lines through modulation of CXC-chemokine/NF- $\mathrm{B}$ signalling pathway. Sci Rep 6: 24675, 2016.

53. Selvendiran K, Ahmed S, Dayton A, Kuppusamy ML, Rivera BK, Kálai T, Hideg K and Kuppusamy P: HO-3867, a curcumin analog, sensitizes cisplatin-resistant ovarian carcinoma, leading to therapeutic synergy through STAT3 inhibition. Cancer Biol Ther 12 $837-845,2011$
54. Chanvorachote P, Pongrakhananon V, Wannachaiyasit S, Luanpitpong S, Rojanasakul Y and Nimmannit U: Curcumin sensitizes lung cancer cells to cisplatin-induced apoptosis through superoxide anion-mediated Bcl-2 degradation. Cancer Invest 27: 624-635, 2009.

55. di Masi A, Leboffe L, De Marinis E, Pagano F, Cicconi L, Rochette-Egly C, Lo-Coco F, Ascenzi P and Nervi C: Retinoic acid receptors: from molecular mechanisms to cancer therapy. Mol Aspects Med 41: 1-115, 2015.

56. Flamini MI, Gauna GV, Sottile ML, Nadin BS, Sanchez AM and Vargas-Roig LM: Retinoic acid reduces migration of human breast cancer cells: role of retinoic acid receptor beta. J Cell Mol Med 18: 1113-1123, 2014.

57. Chen CF, Goyette P and Lohnes D: RARgamma acts as a tumor suppressor in mouse keratinocytes. Oncogene 23: 5350-5359, 2004.

58. Hatoum A, El-Sabban ME, Khoury J, Yuspa SH and Darwiche N: Overexpression of retinoic acid receptors alpha and gamma into neoplastic epidermal cells causes retinoic acid-induced growth arrest and apoptosis. Carcinogenesis 22: 1955-1963, 2001.

59. Shehzad A, Wahid F and Lee YS: Curcumin in cancer chemoprevention: molecular targets, pharmacokinetics, bioavailability, and clinical trials. Arch Pharm (Weinheim) 343: 489-499, 2010.

60. Ramachandran C, Rodriguez S, Ramachandran R, Raveendran Nair PK, Fonseca H, Khatib Z, Escalon E and Melnick SJ: Expression profiles of apoptotic genes induced by curcumin in human breast cancer and mammary epithelial cell lines. Anticancer Res 25: 3293-3302, 2005.

61. Van Erk MJ, Teuling E, Staal YC, Huybers S, Van Bladeren PJ, Aarts JM and Van Ommen B: Time- and dose-dependent effects of curcumin on gene expression in human colon cancer cells. J Carcinog 3: 8, 2004. 